LA-UR- $96-2599$

$$
\text { CONF- } 9608133-1
$$

TITLE: Emittance Growth of a Short Electron Bunch in Circular Motion

A18 28 ings

OSTI

AUTHOR(S): B. E. Carlsten and J. C. Goldstein

SUBMITTED TO: 18th International Free Electron Laser Conference

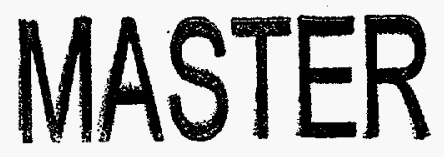

By acceptance of this article, the publisher recognizes that the U.S. Govemment retains a nonexclusive, royalty-free license to publish or reproduce the published form of this contribution, or to allow others to do so, for U.S. Government purposes.

The Los Alamos National Laboratory requests that the publisher identify this article as work performed under the auspices of the U.S. Department of Energy.

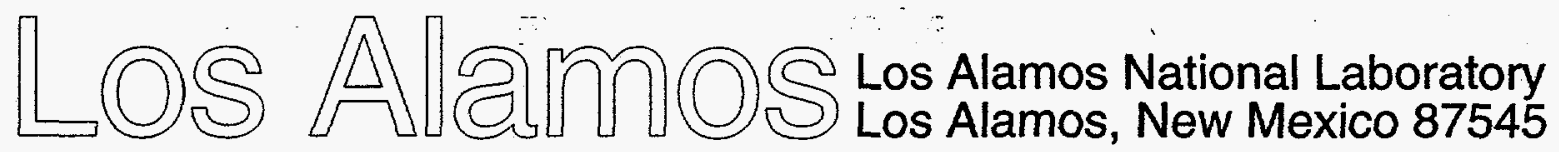




\section{DISCLAIMER}

Portions of this document may be illegible in electronic image products. Images are produced from the best available original document. 


\section{DISCLAIMER}

This report was prepared as an account of work sponsored by an agency of the United States Government. Neither the United States Government nor any agency thereof, nor any of their employees, makes any warranty, express or implied, or assumes any legal liability or responsibility for the accuracy, completeness, or usefulness of any information, apparatus, product, or process disclosed, or represents that its use would not infringe privately owned rights. Reference herein to any specific commercial product, process, or service by trade name, trademark, manufacturer, or otherwise does not necessarily constitute or imply its endorsement, recommendation, or favoring by the United States Government or any agency thereof. The views and opinions of authors expressed herein do not necessarily state or reflect those of the United States Government or any agency thereof. 


\title{
Emittance Growth of a Short Electron Bunch in Circular Motion*
}

\author{
Bruce E. Carlsten ${ }^{1}$ and John C. Goldstein \\ Los Alamos National Laboratory, Los Alamos, NM 87545, USA
}

\begin{abstract}
A short electron bunch undergoing circular motion produces space-charge forces that do not decrease with increasing bunch energy, unlike those induced by straight-line motion. These energy-independent forces can be separated into a noninertial space-charge force and a coherent synchrotron radiation force. These forces result in an energy spread in the bunch, and can lead to a potentially large emittance growth. These effects can take place in both (1) bunch compression systems used to increase the peak current and (2) the wiggler itself. Numerical estimates of the emittance growth in a wiggler for a 1-ps long, 1-mm radius, 1-nC electron bunch can be as large as $0.1 \pi \mathrm{mm}$ mrad per wiggle period; the energy spread can grow as much as $30 \mathrm{keV}$ per wiggle period. These types of beam quality degradation may become significant for future, shortwavelength free-electron lasers requiring high-brightness electron beams, especially for selfamplified spontaneous emission operation.
\end{abstract}

\footnotetext{
* This work was supported by the Los Alamos Laboratory Directed Research and Development program, under the auspices of the U. S. Department of Energy

1 Corresponding author. Tel. +1 505667 5657, fax +1 505667 8207, e-mail bcarlsten@lanl.gov.
} 


\section{Introduction}

Recent work has identified the existence of two space-charge forces that do not scale inversely with the square of the beam energy [1-4]. These forces occur if a short electron bunch is in circular motion; one is known as the noninertial space-charge force and is a short range force, the other is known as the coherent synchrotron radiation force and operates over a longer range and can radiate significant amounts of power. Both forces can cause a significant redistribution of the energy of the individual particles within the bunch.

The component of these forces along the direction of motion can be analytically found for short uniform density lines of charge undergoing circular motion. The total space-charge force for the line in the direction of motion is given by [4]

$$
E_{\theta}=\left.\frac{\lambda}{4 \pi \varepsilon} \frac{1}{r_{r e t}-\vec{r}_{r e t} \cdot \vec{u}_{r e t} / c}\left(\frac{1}{\gamma^{2}}-\beta^{2} \frac{x}{R}+\beta^{2} \frac{r}{R}\left(1-\cos \left(\zeta^{\prime}\right)\right)\right)\right|_{\zeta_{r}} ^{\zeta_{f}}
$$

where $\theta$ is the direction of motion, $R$ is the radius of curvature of the circular motion, $\beta$ is the azimuthal velocity divided by the speed of light $c, \lambda$ is the current density, $x$ is the transverse displacement of the observer location in the bend plane relative to the trajectory of the source line (we can additionally define $y$ to be the displacement in the perpendicular displacement), $\vec{r}_{\text {ret }}$ is the vector from the source point (presently at angular position $\zeta$ ) at the retarded time to the observer location, $r_{r e t}=\left|\vec{r}_{\text {ret }}\right|, \vec{u}_{\text {ret }}$ is the retarded velocity of that point at the retarded time, $\zeta^{\prime}$ is the retarded angle of the source point's azimuthal position, and $\zeta_{f}$ and $\zeta_{r}$ are the present azimuthal angles of the front and rear of the bunch, respectively. Note that $\zeta^{\prime}$ and $\zeta$ are considered positive if they lie behind the observer position. The retarded angular position $\zeta^{\prime}$ in turn must satisfy the transcendental equation

$$
\beta^{2} R^{2}\left(\zeta^{\prime}-\zeta\right)^{2}=\rho^{2}+2 R(R+x)\left(1-\cos \zeta^{\prime}\right)
$$


where now $\rho$ is a total transverse displacement, $\rho=\sqrt{x^{2}+y^{2}}$.

This force along the direction of motion will change the energy of particles within a bunch compressor (used to increase the peak current of the electron bunch) and even within the wiggler itself, destroying the achromaticity of that component, and resulting in a potentially large emittance growth which could degrade the performance of the free-electron laser (FEL). This emittance growth is directly related to the energy spread introduced by these forces.

The emittance growth within large angle dipole fields (from several degrees to tens of degrees), such as the case in bunch compressors, have been calculated before [4]; the thrust of this paper will be the consideration of the degradation of the electron beam from these forces when the beam is in the wiggler. From geometrical reasons, the emittance growth in a wiggle period scales roughly as $\Delta \varepsilon_{w} \sim x_{r m s} \Delta E_{w} \alpha$, where $x_{r m s}$ is the rms bunch length, $\Delta E_{w}$ is the rms energy spread induced by one wiggle period, and $\alpha$ is the angle the beam is bent by the dipole field. For compressors, $\alpha$ is on the order of unity, and a relatively minor increase in the energy spread can result in a large emittance growth. In a wiggler, however, $\alpha \approx K / \gamma$, where now $K$ is the wiggler parameter which is on the order of unity, and the angle is very small. In this case, even a relatively large induced energy spread will not necessarily lead to a substantial emittance growth.

In Figs. 1 and 2 we show the energy spread induced in a 1-ps, 1-nC, 100-MeV electron bunch after passing through two wiggle periods of the self-amplified spontaneous emission (SASE) case we will consider in the following sections, as calculated by the modified accelerator tracking code PARMELA [4,5]. The energy spread in Fig. 1 is calculated from a relatively poor approximation of the coherent synchrotron radiation force alone (Eqn. (2) was used to find the retarded angular position, even if the angle exceeded the maximum wiggle angle); the energy spread in Fig. 2 is calculated from a very good approximation of the noninertial space-charge force alone. The effect from the coherent synchrotron radiation force is over estimated because of causality and the wiggle geometry; the actual induced energy spread is certainly less than this value and has been argued to be in fact negligible [2]. For this nominal case, the emittance growths per 
wiggle period calculated by PARMELA is about $0.012 \pi \mathrm{mm} \mathrm{mrad}$ from the noninertial spacecharge force and about $0.005 \pi \mathrm{mm}$ mrad from the coherent synchrotron radiation force. Note that the instantaneous, or slice, energy spread, and thus emittance growth, is much smaller than the overall energy spread in Fig. 1; the instantaneous energy spread is the same as the overall energy spread in Fig. 2. Previous simulations [6] with the FEL simulation code FELEX [7] have shown that the lasing performance is effected more by an increase in the slice beam quality than the overall integrated beam quality, as long as the beam quality over the full slippage distance as the bunch travels through the wiggler is comparable to the slice beam quality. In this paper we will only consider the degradation of the beam quality from the noninertial space-charge force; it should be larger than the degradation from the coherent synchrotron radiation. It should be noted that this may also be the case for extremely high energies. Although the rms bunch radius will become very small, decreasing the effect from the noninertial space-charge force, the suppression of the retarded angle will become larger, decreasing also the effect from the coherent synchrotron radiation force.

Because the emittance growth and induced energy spread in a wiggler from the noninertial space-charge force is proportional to the number of wiggle periods (the effect from each wiggle period is correlated), this effect will be most important for SASE amplifiers, with hundreds or even thousands of wiggle periods. The integrated effect after 500 wiggle periods for the above example from the noninertial space-charge force would be $2.5 \pi \mathrm{mm}$ mrad and the energy spread would be $6 \%$. The effect of the energy spread in this example is clearly more detrimental to the lasing.

The following two sections in this paper will explore the issues outlined above. In the first section, we will compare the total power radiated by the coherent synchrotron radiation for uniform circular motion to the power radiated by SASE saturated lasing, for a nominal example with a 2-cm wiggle period and a $100-\mathrm{MeV}$ beam energy. The coherent synchrotron radiation will dominate the lasing power, thereby making it reasonable to expect that the emittance growth and induced energy spread from these newly understood space-charge forces will dominate the emittance growth and induced energy spread due to the actual FEL mechanism. In the next section, the energy spread and emittance growth scalings from the noninertial space-charge force will be presented, in terms 
of the bunch radius, the bunch length, the bunch energy, the wiggle period, and the normalized wiggle parameter $K$.

\section{Total Radiated Power}

In this section we will compare the total radiated power of both the coherent synchrotron radiation (from equivalent uniform circular motion) and the SASE lasing, for a $100-\mathrm{MeV}, 1-\mathrm{mm}$ radius, 1-ps long (FWHM), 1-nC electron bunch in a wiggler with a period $\lambda_{w}$ of $2 \mathrm{~cm}$, and with a normalized wiggle parameter $K=e \lambda_{w} B_{p} / 2 \pi m c$ of unity, where now $B_{p}$ is the peak wiggler magnetic field, $m$ is the electronic mass, and $e$ is the electronic charge. The actual coherent synchrotron radiation over the entire length of the wiggler is much less than that for uniform circular motion over a single arc with equal path length; however this calculation will demonstrate that the space-charge forces leading to the coherent synchrotron radiation and the noninertial spacecharge effect are large enough to be of concern.

We will first estimate the SASE saturated power and number of required wiggle periods, using [8]. The efficiency of the radiation (radiated power divided by beam power) is given by the dimensionless parameter $\rho$ and the number of required wiggle periods to reach saturation is given by $1 / \rho$, where

$$
\rho^{3}=\frac{e K^{2}[J J]^{2} Z_{o} j \lambda_{w}^{2}}{128 \pi^{2} \gamma^{3} m c^{2}}
$$

and where the normalized mass $\gamma=197$ for this example, $Z_{o}=377 \Omega$, the current density $j=10^{9} / \pi \mathrm{A} / \mathrm{m}^{2}$, and the Bessel function parameter $[J J]=J_{o}(\xi)-J_{1}(\xi)$, where now $\xi=K^{2} / 4\left(1+K^{2} / 2\right)$. For this example, $\rho=2.01\left(10^{-3}\right)$; the saturated power is about $0.2 \%$ of the beam power, and about 500 wiggle periods are required. This example is not ridiculous; the resonant light wavelength is about $500 \mathrm{~nm}$, and the slippage over the entire wiggler is about 0.25 $\mathrm{mm}$, somewhat smaller than the bunch FWHM. The peak wiggler magnetic field is $0.54 \mathrm{~T}$. 
For continuous circular motion (not interrupted by wiggling back and forth), the coherent synchrotron radiated power is given by $[1,2]$

$$
P=3^{2 / 3} \frac{N^{2} e^{2} \beta c}{4 \pi \varepsilon R^{2 / 3} \delta^{4 / 3}}
$$

where $N$ is the number of electrons in the bunch and $\delta$ is the rms bunch length. The maximum wiggle angle is given by $K / \gamma$, and for this case is $5.1 \mathrm{mrad}$, leading to a bend radius of $R=0.98 \mathrm{~m}$. Using this expression, we find that the average energy loss per electron is about 2.8 $\mathrm{MeV} / \mathrm{m}$, or $60 \mathrm{keV}$ per wiggle period. The numerically calculated average energy loss per wiggle period (corresponding to the simulation shown in Fig. 1) is $120 \mathrm{keV}$ in a length corresponding to a single wiggle period, in reasonable agreement with the analytic estimate. The energy loss from the coherent synchrotron radiation force in a wiggler is certainly less than this value (geometric arguments show that it is a factor of $\lambda_{w}^{3} / 192 \delta R^{2}$ smaller) - however, the energy spread induced by the noninertial space charge force will be of a comparable size even when the beam is wiggling. Note that in $10 \mathrm{~m}$ (equivalent to 500 wiggle periods), an energy spread on the order of a tenth of the total beam energy will be generated by this mechanism.

\section{Emittance growth scaling from the noninertial emittance growth force}

In this section, we will present plots of the normalized, rms emittance growth and the rms energy spread growth as various parameters are changed. We will then present scaling formulas that apply over the parameter range.

In Figs. 3-7, the beam and wiggler parameters are those outlined in the previous section. In all figures, we will plot both the normalized emittance growth and the energy spread growth from the noninertial space-charge force per wiggle period.

In Fig. 3, 4, 5, 6, and 7, these quantities are plotted versus bunch radius, FWHM bunch length, beam energy, wiggle period, and wiggler parameter $K$, respectively. 
The normalized, rms emittance growth induced per wiggle period over this parameter range is given roughly by (the scalings are from Eqn. (1))

$$
\Delta \varepsilon_{w} \approx \frac{a^{2} K^{2}}{\delta^{2}}\left(\frac{100}{E}\right)^{2} 0.012 \pi \mathrm{mm} \mathrm{mrad}
$$

where the FWHM bunch radius $a$ is in mm, the FWHM bunch length $\delta$ is in ps, and the beam energy $E$ is in $\mathrm{MeV}$.

The rms energy spread induced per wiggle period is likewise given by

$$
\Delta E_{w} \approx \frac{a K}{\delta^{2}}\left(\frac{100}{E}\right) 12 \mathrm{keV}
$$

where now the wiggle period $\lambda_{w}$ is given in $\mathrm{cm}$. Eqns. (5) and (6) agree reasonably well with Figs. 3-7. Using Eqn. (3), we can estimate the emittance and energy spread growth scalings over the entire wiggler, for SASE operation:

$$
\begin{aligned}
& \Delta \varepsilon_{\text {total }} \sim \frac{a^{8 / 3} K^{4 / 3}}{\delta^{5 / 3} \lambda_{w}^{2 / 3} E} \\
& \Delta E_{\text {total }} \sim \frac{a^{5 / 3} K^{1 / 3}}{\delta^{5 / 3} \lambda_{w}^{2 / 3}}
\end{aligned}
$$

Note that there is a hidden scaling of the energy is these expressions - from the resonance equation, $1 / \lambda_{w} \approx 1 /\left(\gamma^{2} \lambda_{l}\right)$, where $\lambda_{l}$ is the wavelength of the radiated light.

The emittance growth can be made negligible rather quickly by decreasing the bunch radius and $K$ (although at the cost of higher beam energy) - the energy spread growth requires a significantly higher beam energy to be comparably reduced.

As a final example of this effect, consider the 1-GeV, FEL proposed for TESLA [9]. The rms beam radius is $0.05 \mathrm{~mm}$, rms bunch length is $0.05 \mathrm{~mm}$, bunch charge is $1 \mathrm{nC}, K$ is 1.26 , the 
wiggle period is $27.3 \mathrm{~mm}$, and there are about 1100 wiggle periods. By direct PARMELA simulation, the emittance growth induced per wiggle period is found to be only about $10^{-5} \pi \mathrm{mm}$ mrad (which is negligible even integrated over the entire wiggler) and the rms energy spread induced per wiggle period is found to be about $1.3 \mathrm{keV}$, or about $1.5 \mathrm{MeV}$ over the length of the entire wiggler ( $0.15 \%$ energy spread). Note that Eqn. (5) predicts an emittance growth of about $6\left(10^{-6}\right) \pi \mathrm{mm} \mathrm{mrad}$, and Eqn. (6) predicts an energy spread growth of $0.7 \mathrm{keV}$ per wiggle period, both in reasonable agreement with the simulations.

\section{Conclusion}

We have presented a numerical analysis of the rms emittance growth and rms energy spread induced by the noninertial space-charge force in a wiggler. Because of the small angle of the wiggling, the induced energy spread will probably be more detrimental to the lasing performance. Empirical formulas were given for the emittance and energy spread growth both for a single wiggle period and for an entire wiggler required for SASE operation. The effect studied in this paper will lead to somewhat higher beam energies and longer wiggler lengths than previously thought, for future short wavelength, SASE experiments.

\section{References}

1. J. S Nodvick and D. S. Saxon, "Suppression of coherent radiation by electrons in a synchrotron," Phys. Rev., 96, p. 180 (1954).

2. Ya. S. Derbenev, J. Rossbach, E. L. Saldin, and V. D. Shiltsev, "Microbunch radiative tailhead interaction," TESLA-FEL report 95-05 (1995).

3. B. E. Carlsten and T. O. Raubenheimer, "Emittance growth of bunched beams in bends," Phys. Rev. E, 51, p. 1453 (1995). 
4. B. E. Carlsten, "Calculation of the noninertial space-charge force and the coherent synchrotron radiation force for short electron bunches in circular motion using the retarded Green's function technique," to be published in Phys. Rev. E, July 1995.

5. L. Young, Los Alamos National Laboratory, private communication.

6. B. E. Carlsten, J. C. Goldstein, E. J. Pitcher, and M. J. Schmitt, "Simulations of APEX accelerator performance in the new nonthermalized photoinjector regime," Nucl. Instrum. Meth. Phys. Res., A331, p. 307 (1993).

7. B. D. McVey, Nucl. Instrum. Meth. Phys. Res., A250, p. 449 (1986).

8. K.-J. Kim, "An analysis of self-amplified spontaneous emission," Nucl. Instrum. Meth. Phys. Res., A250, p. 396 (1986).

9. Deutsches Elektronen-Synchrotron, DESY, "A VUV free electron laser at the TESLA test facility at DESY - conceptual design report," TESLA-FEL report 95-03 (1995).

\section{Figure Captions}

1. Energy-phase plot showing energy spread induced in two wiggle periods by the coherent synchrotron radiation force.

2. Energy-phase plot showing energy spread induced in two wiggle periods by the noninertial space-charge force.

3. Emittance and energy spread induced in one wiggle period as a function of bunch radius.

4. Emittance and energy spread induced in one wiggle period as a function of bunch length.

5. Emittance and energy spread induced in one wiggle period as a function of beam energy. 
6. Emittance and energy spread induced in one wiggle period as a function of wiggle period.

7. Emittance and energy spread induced in one wiggle period as a function of $K$ 


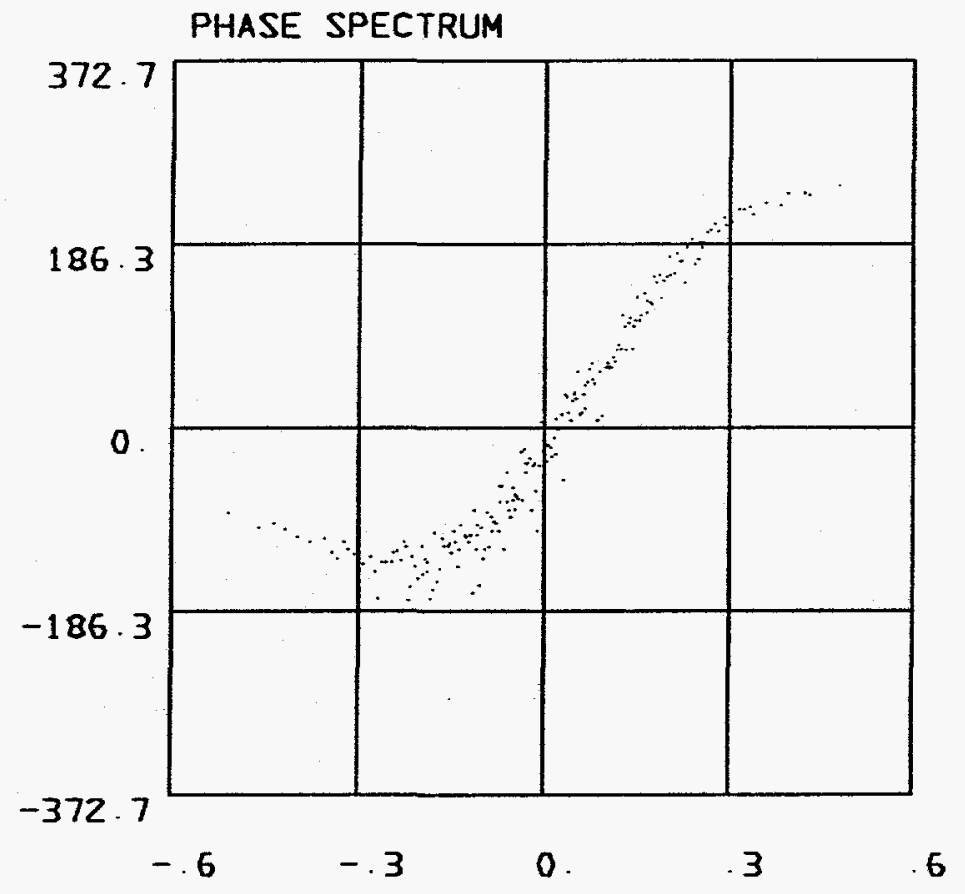

E-ES VS. PHI-PHIS

$E S=99.724 . P S=140.43, Z=7.2$

Fig 1 


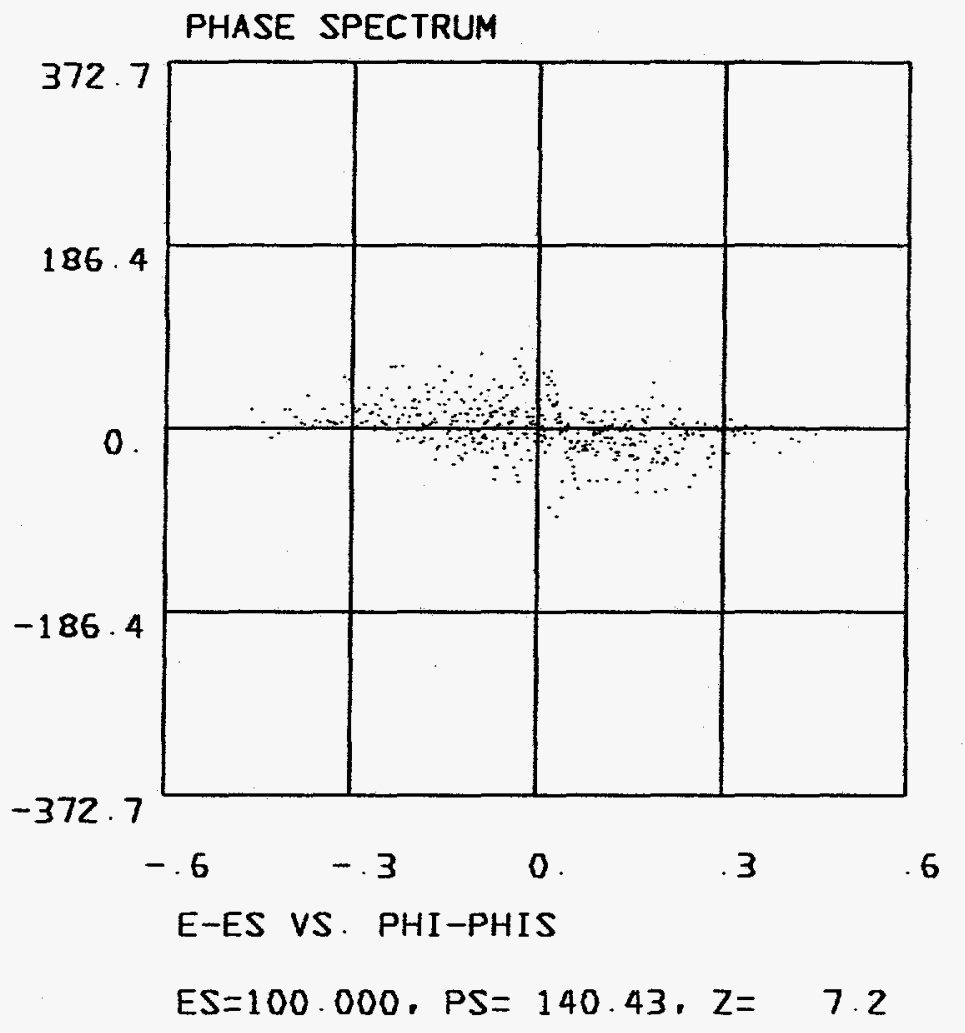

$\operatorname{Fig} 2$ 


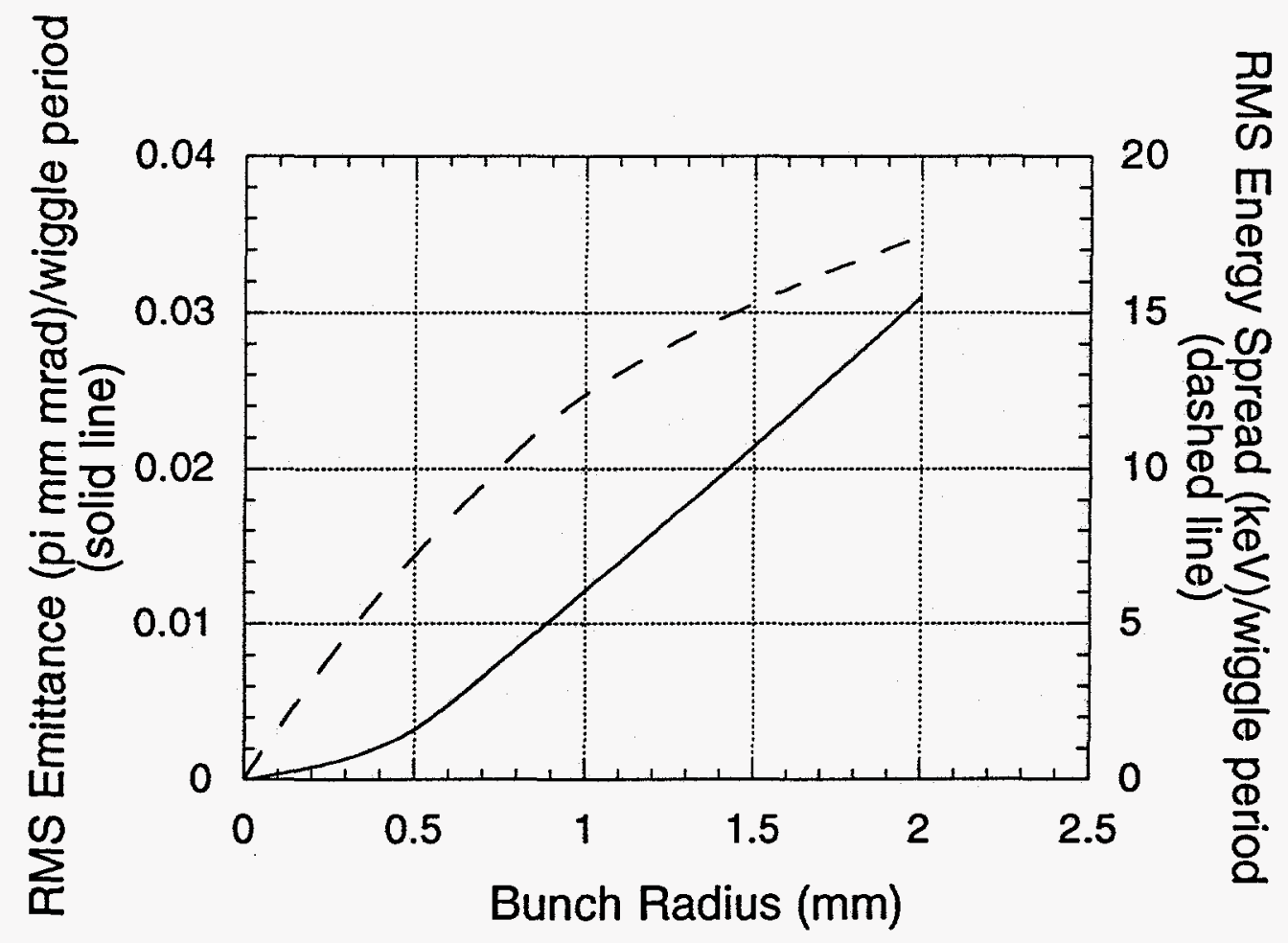

Fig 3 


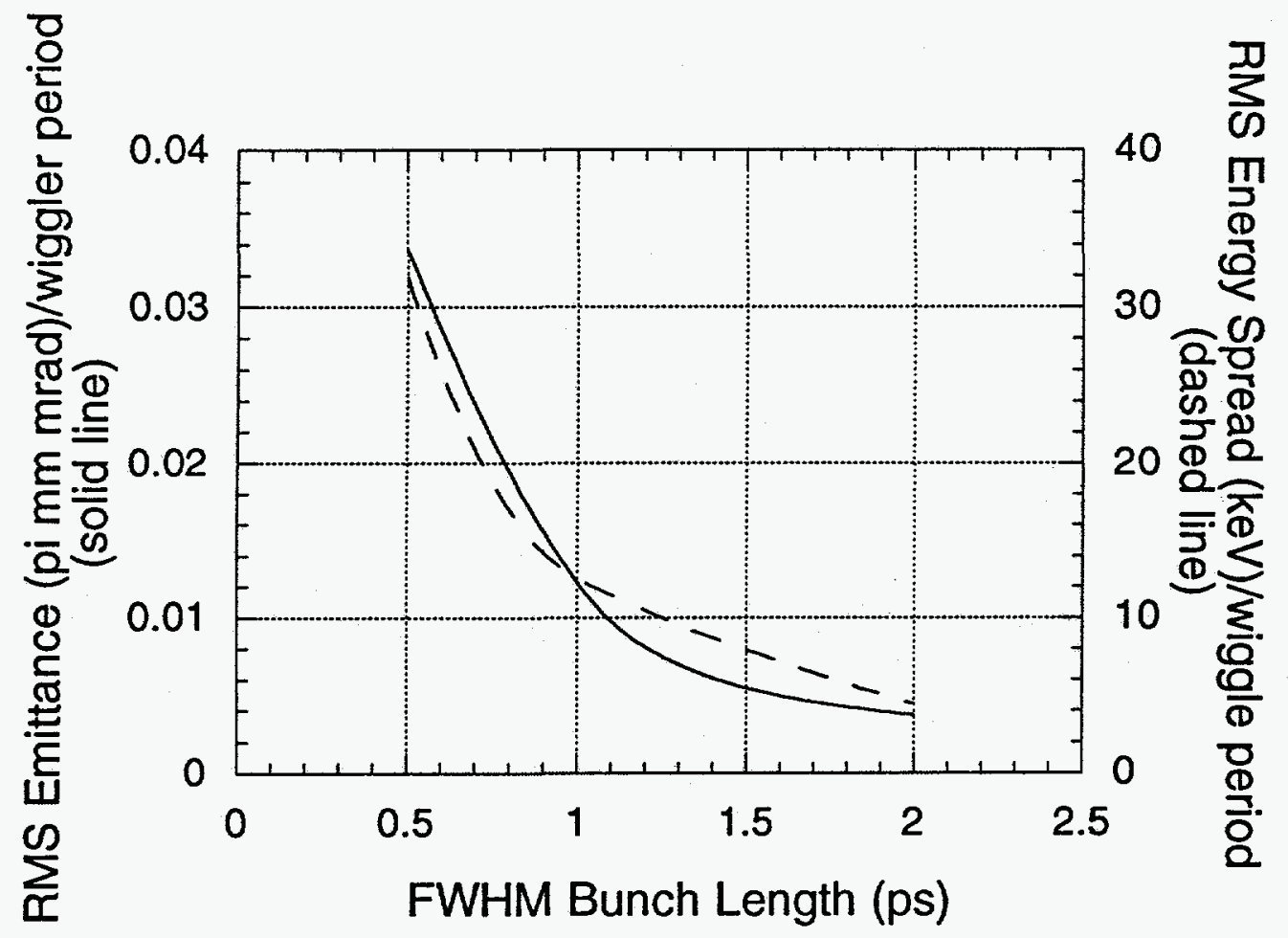

Fig4 


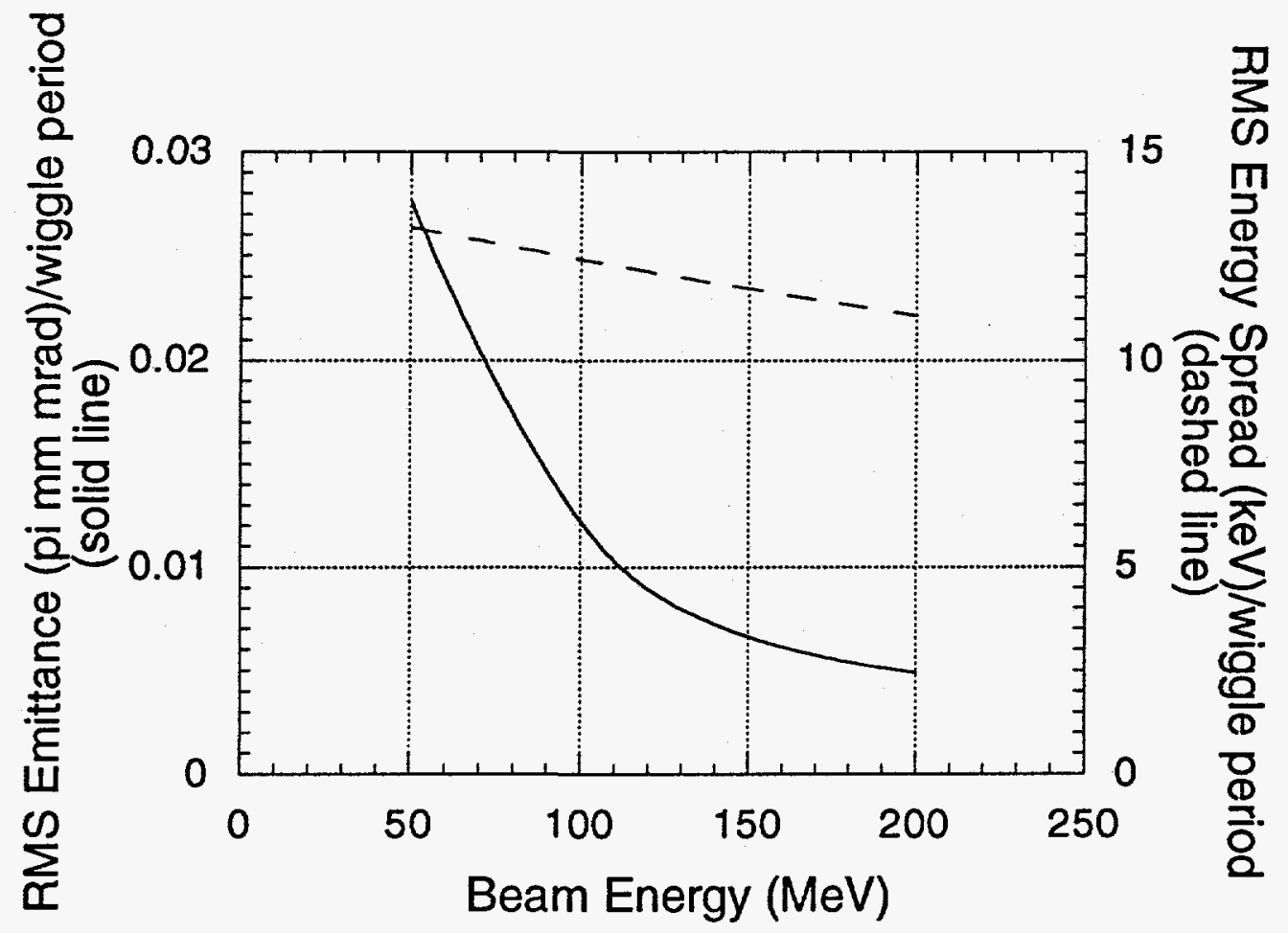

Figs 


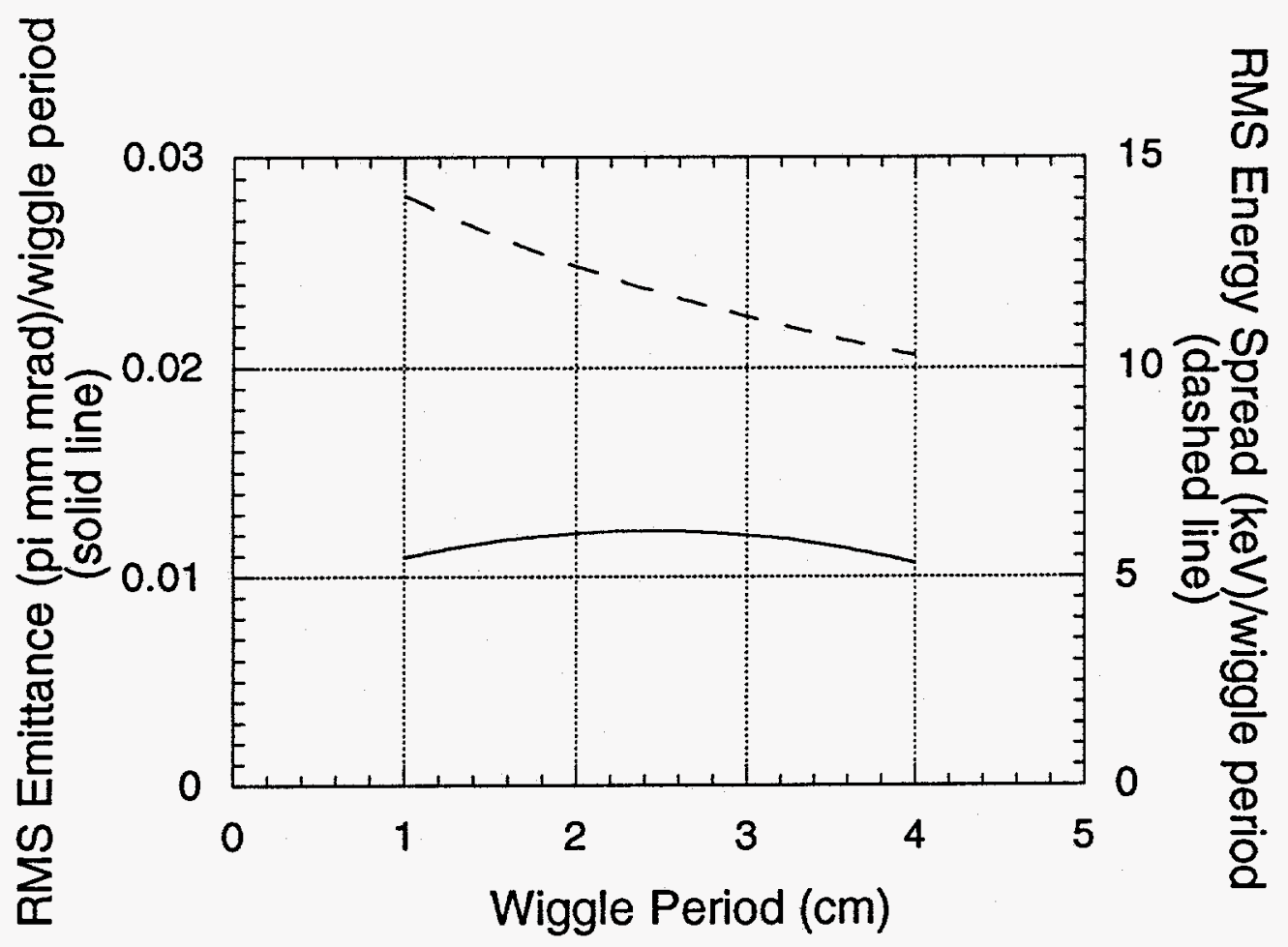

Figl 


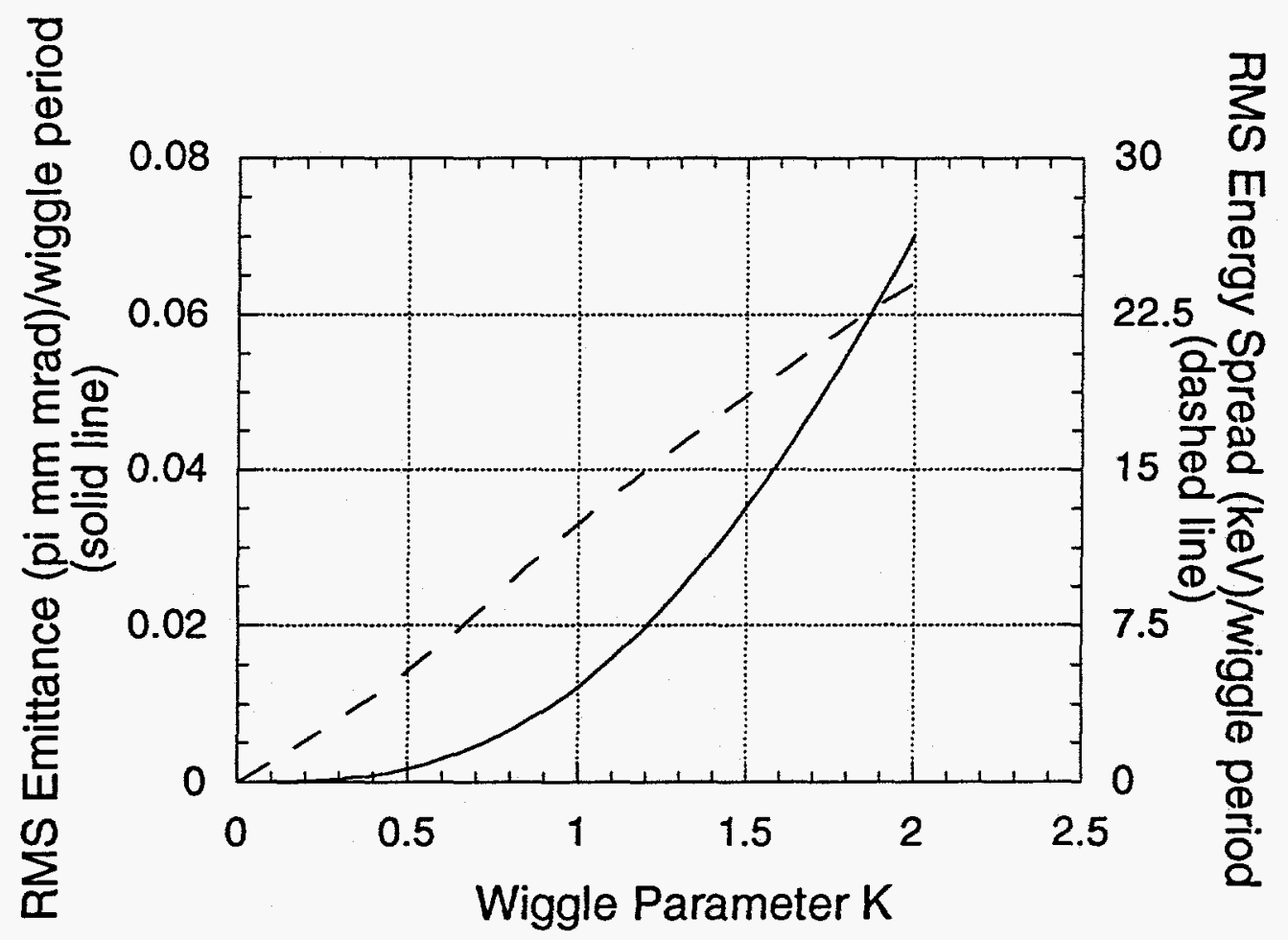

Fig7 